\title{
Physical Origins of Extreme Cross-Polarization Extinction in Confocal Microscopy
}

\author{
Meryem Benelajla $\odot,{ }^{1,2}$ Elena Kammann, ${ }^{1}$ Bernhard Urbaszek $\odot,{ }^{2}$ and Khaled Karrai ${ }^{1, *}$ \\ ${ }^{1}$ attocube systems AG, Eglfinger Weg2, 85540 Haar, Germany \\ ${ }^{2}$ Université de Toulouse, INSA-CNRS-UPS, LPCNO, Toulouse 31077, France
}

(Received 29 April 2020; revised 14 January 2021; accepted 15 February 2021; published 7 April 2021)

\begin{abstract}
Confocal microscopy is an essential imaging tool for biological systems, solid-state physics and nanophotonics. Using confocal microscopes allows performing resonant fluorescence experiments, where the emitted light has the same wavelength as the excitation laser. These challenging experiments are carried out under linear cross-polarization conditions, rejecting laser light from the detector. In this work, we uncover the physical mechanisms that are at the origin of the yet-unexplained high polarization rejection ratio which makes these measurements possible. We show in both experiment and theory that the use of a reflecting surface (i.e., the beam splitter and mirrors) placed between the polarizer and analyzer in combination with a confocal arrangement explains the giant cross-polarization extinction ratio of $10^{8}$ and beyond. We map the modal transformation of the polarized optical Gaussian beam. We find an intensity "hole" in the reflected beam under cross-polarization conditions. We interpret this hole as a manifestation of the Imbert-Fedorov effect, which deviates the beam depending on its polarization helicity. This result implies that this topological effect is amplified here from the usually observed nanometer to the micrometer scale due to our cross-polarization dark-field methods. We confirm these experimental findings for a large variety of commercially available mirrors and polarization components, allowing their practical implementation in many experiments.
\end{abstract}

DOI: 10.1103/PhysRevX.11.021007

Subject Areas: Nanophysics, Optics, Photonics

\section{INTRODUCTION}

In optical spectroscopy experiments, it is crucial to excite an emitter with a laser very close to its transition energy. Experiments under resonant excitation are essential for accessing the intrinsic optical and spin-polarization properties of a large class of emitters [1-5]. Resonance fluorescence is not limited to quantum dots [6], which are known as robust, fast, bright, and narrow-linewidth emitters of single photons $[7,8]$. It is also used to study novel solid-state material systems with longer coherence times suitable for quantum information storage, computation, and sensing at a single-photon level [9-11] such as transition metal dichalcogenide monolayers [12], rare earth elements [13], single nitrogen vacancy [10], tin vacancy [14], silicon [15], and germanium vacancy in diamond [16]. Using linear crosspolarization in a confocal setup has been successfully employed as a dark-field method to carry out resonant fluorescence experiments to suppress scattered laser light, with the added benefit of high spatial resolution [17,18].

\footnotetext{
*khaled.karrai@attocube.com
}

Published by the American Physical Society under the terms of the Creative Commons Attribution 4.0 International license. Further distribution of this work must maintain attribution to the author(s) and the published article's title, journal citation, and DOI.
Resonant fluorescence experiments allow crucial insights into light-matter coupling, such as the interaction of a singlephoton emitter with its environment [19], with optical cavities [20], and also studying single defects in atomically thin materials such as $\mathrm{WSe}_{2}$ [12]. Dark-field confocal techniques allow developing single-photon sources with high degrees of photon indistinguishability [21-23] and longer coherence [24]. In practice, dark-field laser suppression is not just a spectroscopy tool; it is also a key part of more matured quantum technology systems [25].

Resonant excitation is absolutely necessary for manipulating quantum states or for any operation that relies on phase coherence. A striking example is the realization and verification of quantum entanglement between an $\mathrm{NV}$ electron spin qubit and a telecom-band photonic qubit [26]. Here, readout via a phonon sideband is impossible, as phonon emission implies that the target states are no longer entangled. The dark-field approach is adopted for enabling practicable quantum networks using quantum dots $[20,27]$ and diamond defects $[9,11,28]$ embedded in optical cavities.

Despite many advances based on experiments in confocal microscopes with cross-polarization laser rejection, the physical mechanisms that make these experiments possible are not well understood, hampering further progress in this field. The key figure of merit is the suppression of the excitation laser background by at least 6 orders of magnitude. Pioneering work by Wilson et al. shows that 
cross-polarization extinction of a confocal microscope is much higher than of a classical microscope and that it could reach, but never exceed, the polarizer intrinsic extinction limitation, which in their case is $10^{4}$ [29] to $10^{5}$ [30]. This result is very different from our findings, in which we demonstrate how to go beyond the intrinsic polarizer extinction limit by 3-4 orders of magnitude. Indeed, a suppression by a factor of $10^{8}$ [31] up to $10^{10}$ (this work) have been measured. But this result is very surprising, as not only real polarizer extinction limitation but also the lenses, mirrors, and beam splitter in such a system reduce the theoretical extinction limit to the $10^{3}-10^{4}$ range.

In this work, we explain the physics behind the giant enhancement of the extinction ratio by up to 7 orders of magnitude that make microscopy based on dark-field laser suppression possible. The measurements of resonant fluorescence are typically performed in an epifluorescence geometry [31], for which laser excitation and fluorescence collection are obtained through the same focusing lens. This measurement involves necessarily the use of a beam splitter orienting the backreflected light containing the fluorescence toward a detection channel. In our work, we identify two key ingredients that explain the giant amplification of the cross-polarization extinction ratio: (i) a reflecting surface (i.e., the beam splitter) placed between a polarizer and analyzer and (ii) a confocal arrangement. We demonstrate giant extinction ratios in our experiments for different mirrors (silver, gold, dielectric, and beam-splitter cubes) and polarizers (Glan-Taylor and nanoparticle thin films). We demonstrate that behind this general observation lies the intriguing physics of the Imbert-Fedorov effect $[32,33]$, which deviates a reflected light beam depending on its polarization helicity. We discover that a confocal arrangement amplifies the visibility of the ImbertFedorov effect dramatically, taking it from the nanometer to the micrometer scale. As a result of this micrometer shift, the cross-polarized laser beam is not coupled into the detection fiber, explaining the near-complete suppression of the laser background signal. In other words, we cannot treat the spatial and polarization properties of light separately in our dark-field confocal microscope analysis. In addition to new developments in dark-field microscopy, our experiments provide powerful tools for research in topological photonics [34-36], in the broader context of what is termed the spin-Hall effect of light [36].

In our work, we setup a robust, reproducible experiment and derive a formalism to investigate these remarkable effects at the crossroads of quantum optics and topological photonics.

The paper is structured as follows. In Sec. II, we introduce the experimental setup. Cancellation of polarization leakage is measured and discussed in a first simplified model in Sec. III. The modal transformation of a reflected Gaussian beam is analyzed in Sec. IV. The effect of confocal filtering is discussed in Sec. V.

\section{CONFOCAL MICROSCOPE SETUP}

We use a simplified confocal arrangement as depicted in Figs. 1(a) and 1(b) in order to focus on the most relevant physics leading to extreme laser rejection. A diode laser beam (1) at $\lambda=905 \mathrm{~nm}$ wavelength is launched into a single-mode fiber (2). The light emerges from the $4^{\circ}$ angled flat-polished end with a nearly perfect Gaussian beam with $\omega_{0}=2.5 \mu \mathrm{m}$ mode waist radius at $1 / e^{2}$ of the maximum intensity. A diffraction-limited microscope objective (3) of numerical aperture NA $=0.25$ and focal length of $f=$ $26 \mathrm{~mm}$ focused on the fiber end collimates the light into a 3-mm-waist-radius Gaussian beam. We choose the NA to be significantly larger than the diverging beam half-angle out of the fiber in order to preserve the Gaussian quality of the beam.

A pair of mirrors (4 and 5) mounted on two axis tilt stages allows for fine steering of the collimated beam axis. Next, the beam travels to a linear polarizer (6) mounted on a piezoelectric stepping stage rotating with $20 \mu \mathrm{rad}$ resolution around the optical axis. The best-quality commercial linear polarizers we use for this experiment show an extinction in direct cross-polarization limited to $10^{5}$ for a nanoparticle thin film polarizer and to $10^{6}$ for GlanThomson crystal polarizers. The beam travels then toward a mirror (8), the key element of this experiment, either by passing first through an analyzing polarizer [Fig. 1(a)] for the control measurement or by passing through the analyzer after a reflecting surface for the test experiment [Fig. 1(b)]. We mount the analyzer also on a piezo stepper fine rotation stage. The mirror (9) mounted on the two-axis piezo controlled tilt stage steers the beam into a microscope objective (10) identical to (3) focusing the light into the core of a single-mode fiber (11) identical to (2) allowing for Gaussian $\mathrm{TEM}_{00}$ modal confocal filtering and optical detection [(12)-Si-photodiode] at the other end of the 5-m fiber cable. This confocal arrangement simulates the essential components of the resonant fluorescence confocal microscopes. The reflecting surface plane (8) at $45^{\circ}$ of incidence defines the standard $p$ and $s$ states of polarization with projections along $\vec{e}_{x}$ and $\vec{e}_{y}$, respectively. The reflecting test surfaces in position (8) in Figs. 1(a) and 1(b) we use in this work are commercial protected silver, aluminum, and dielectric high-reflectivity Bragg mirrors and evaporated gold film, as well as nonpolarizing beam-splitter cubes. All such reflecting surfaces are typically used in diffraction-limited confocal microscopes. The results are qualitatively very similar for all these reflecting surfaces. We choose to show here the data measured with silver mirrors only, with the exception of data measured for comparison on a glass surface reflecting from air as discussed at the end of this publication.

We now discuss the measurements in the configuration shown in Fig. 1(b), for which the reflecting test surface is sandwiched between the polarizer and the analyzer. First, 

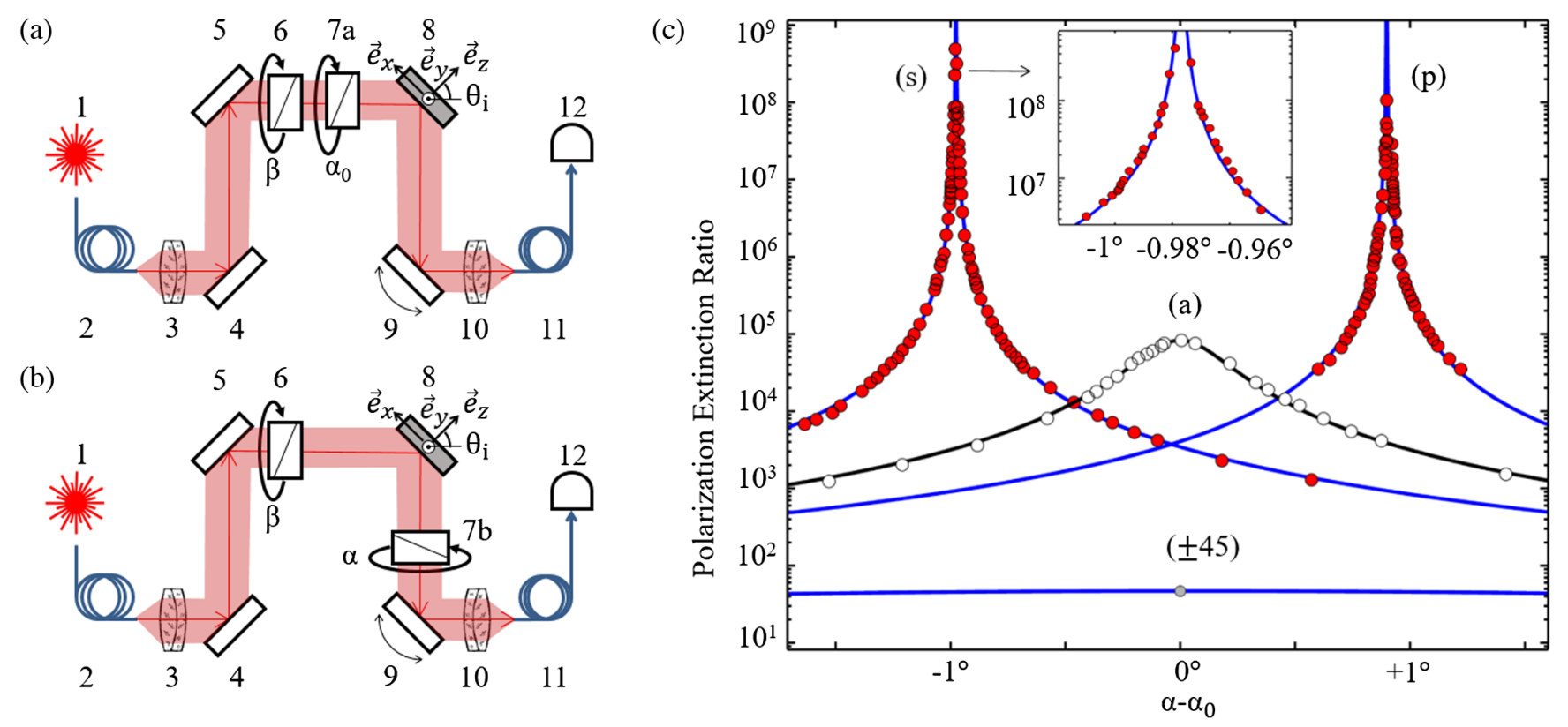

FIG. 1. (a),(b) Cross-polarization extinction setup in a confocal microscope arrangement setup as described in the text. (c) Measured and modeled linear polarization extinction ratio for both $p$ - and $s$-polarized beams around cross-polarization conditions obtained by placing the analyzer before (a) and after (b) a protected silver mirror. A giant extinction enhancement of more than 3 orders of magnitude is obtained in configurations $s$ and $p$ with the reflecting surface placed between crossed polarizers. The inset is a magnification of the polarization extinction near maximum for the $s$ polarization. An angular shift of the location of maxima of extinction is systematically found for the $s$ and $p$ polarization with respect to the reference. When the linear polarization is tilted at $\pm 45^{\circ}$ from the plane of incidence, the extinction reduces dramatically down to about 41 as shown with the lowest curve and data point.

the polarizer angle $\beta$ is adjusted near 0 or $\pi / 2$, for $p$ or $s$ polarization, respectively, while setting the analyzer angle $\alpha$ near cross-polarization at $\beta \pm \pi / 2$. Then, the polarizer and analyzer are subsequently finely rotated to reach maximum extinction at values $\beta$ and $\alpha$, respectively. Once the optimization is reached, $\beta$ remains untouched and the analyzer in its rotator is subsequently placed before the reflecting surface just after the polarizer for our control extinction measurement [Fig. 1(a)]. The analyzer angle must then be adjusted to a new value $\alpha_{0}$ in order to recover maximum nominal extinction specification inherent to the polarizers; $\alpha_{0}$ defines then the $p$ or $s$ reference. The extinction data measured as a function of the analyzer angle $\alpha$ in reference to $\alpha_{0}$ are shown in Fig. 1(c) for the control measurement [Fig. 1(a)] as well as for the $p$ and $s$ polarizations in the configuration [Fig. 1(b)]. Two striking observations stand out. (i) For all the tested reflectors indicated above, the extinction ratio obtained this way is enhanced beyond the $10^{8}$ range when the test mirror surface is sandwiched between the polarizer and the analyzer, reducing this way significantly the polarization leakage of the polarizers. (ii) The analyzer angle for maximum extinction is shifted away from $\alpha_{0}$ by $+0.898^{\circ}$ and $-0.977^{\circ}$ for the $p$ and $s$ polarization, respectively, a significant angular deviation given our resolution of about $10^{-3} \mathrm{deg}$. In the next section, we provide a first explanation for these two striking observations.

\section{CANCELLATION OF POLARIZATION LEAKAGE}

Intuitively, the significant reduction of the polarization leakage field must find its root in a destructive interference effect. The first challenge toward finding an answer to our problem is to offer a model of the polarization leakage. In order to determine the light field at various planes such as after the polarizers and mirrors, we define a right-hand coordinate system $\vec{p}, \vec{s}$ transverse to the optical beam propagation axis $\vec{p} \times \vec{s}$ according to the definition of $p$ and $s$ polarization with respect to the plane of incidence with the test surface (8) in Fig. 1(b). For clarity, $\vec{s} \equiv \vec{e}_{y}$ is perpendicular to the incidence plane. In this section, we test first the simplistic idea that the collimated laser beam can be approximated by a plane wave. We use a Jones matrices formalism projecting the field components along $\vec{p}, \vec{s}$ after each relevant optical element, namely, the matrix $\overline{\bar{P}}(\beta)$ of the polarizer, $\overline{\bar{M}}$ of the reflecting test surface, and $\overline{\bar{A}}(\alpha)$ that of the analyzer. In this formalism, an ideal linear polarizer along $\vec{p}$ and $\vec{s}$ is represented by

$$
\overline{\bar{P}}_{p_{0}}=\left[\begin{array}{ll}
1 & 0 \\
0 & 0
\end{array}\right], \quad \overline{\bar{P}}_{s_{0}}=\left[\begin{array}{ll}
0 & 0 \\
0 & 1
\end{array}\right] .
$$

We assume now that a real physical linear polarizer along $\vec{p}$ or $\vec{s}$ represented by $\overline{\bar{P}}_{p}=\overline{\bar{L}}_{p_{0}}$ and $\overline{\bar{P}}_{s}=\overline{\bar{L}} \overline{\bar{P}}_{s_{0}}$, 
respectively, and is characterized by a polarizer leakage Jones matrix $\overline{\bar{L}}$. The assumption we are making about the physical origin of the leakage is that it is due to lossless coherent scattering such as Rayleigh scattering inclusions in the crystal. This assumption, which we verify experimentally, is that the leakage should be invariant upon an arbitrary angular rotation $\varphi$ around the optical axis $\vec{p} \times \vec{s}$, namely, $\overline{\bar{L}}=\overline{\bar{R}}(\varphi) \overline{\bar{L}} \overline{\bar{R}}(-\varphi)$, where the rotation matrix $\overline{\bar{R}}(\varphi)$ is given by

$$
\overline{\bar{R}}(\varphi)=\left[\begin{array}{cc}
\cos \varphi & -\sin \varphi \\
\sin \varphi & \cos \varphi
\end{array}\right] .
$$

We choose to represent the polarization leakage by a matrix generating a residual elliptical polarization:

$$
\overline{\bar{L}}=\left[\begin{array}{cc}
a & -i b \\
i b & a
\end{array}\right]
$$

where $a^{2}+b^{2}=1$. Such a form is invariant upon rotation. For a high-quality commercially available linear polarizer, $a^{2} \gg b^{2}$, which is the case in our setup, since from our experiment we determine $a^{2} / b^{2} \cong(1.5 \pm 0.5) \times 10^{5}$. This result is the measured nominal leakage seen in Fig. 1(c). We note that the formalism can also be extended to circular polarizers, in which case $a^{2} \cong b^{2}$ and the leakage stems from the slight difference between the two terms.

We assume an incoming laser field $\vec{E}_{p}$ initially $p$ polarized that we rotate at an angle $\beta$, aligning it with the polarizer such $\vec{E}(\beta)=\overline{\bar{R}}(\beta) \vec{E}_{p}$. This field first traverses the leaky polarizer also rotated at $\beta$ such that $\overline{\bar{P}}(\beta)=$ $\overline{\bar{R}}(\beta) \overline{\bar{L}}_{\bar{P}} \overline{\bar{R}}_{0}(-\beta)$ followed by the mirror matrix $\overline{\bar{M}}$ and by the analyzer matrix rotated at an angle $\alpha$, namely, $\overline{\bar{A}}(\alpha)=\overline{\bar{R}}(\alpha) \overline{\bar{L}} \overline{\bar{A}}_{p_{0}} \overline{\bar{R}}(-\alpha)$, so the field $\vec{E}$ just after the analyzer is written

$$
\vec{E}=\overline{\bar{A}}(\alpha) \overline{\bar{M}} \overline{\bar{P}}(\beta) \overline{\bar{R}}(\beta) \vec{E}_{p} .
$$

The mirror Jones matrix for a plane wave is written

$$
\overline{\bar{M}}=\left[\begin{array}{cc}
r_{p} & 0 \\
0 & r_{s}
\end{array}\right]
$$

where $r_{p, s}$ are the complex-valued Fresnel reflectivity coefficients $r_{p}=\left(\tilde{\epsilon} \cos \theta_{i}-\sqrt{\tilde{\epsilon}-\sin ^{2} \theta_{i}}\right) /\left(\tilde{\epsilon} \cos \theta_{i}+\sqrt{\tilde{\epsilon}-\sin ^{2} \theta_{i}}\right)$ and $r_{s}=\left(\cos \theta_{i}-\sqrt{\tilde{\epsilon}-\sin ^{2} \theta_{i}}\right) /\left(\cos \theta_{i}+\sqrt{\tilde{\epsilon}-\sin ^{2} \theta_{i}}\right)$ [37], where the test surface material enters through its complex-valued effective dielectric function $\tilde{\epsilon}=n^{2} / \epsilon_{\text {back }}$ or, equivalently, its optical constant $n^{2}=\epsilon_{1}+i \epsilon_{2}$, which is tabulated for noble mirror metals [38]. Here, $\epsilon_{\text {back }}$ is the dielectric function of the medium covering the mirror. Silver and aluminum mirrors are often covered with about $100 \mathrm{~nm}$ of
$\mathrm{SiO}_{2}$ protective layer. After a lengthy but straightforward calculation, we determine the light intensity just after the analyzer:

$$
\begin{aligned}
I= & a^{2}\left|r_{p} \cos \alpha \cos \beta+r_{s} \sin \alpha \sin \beta\right|^{2} \\
& +b^{2}\left|r_{p} \cos \alpha \sin \beta-r_{s} \sin \alpha \cos \beta\right|^{2} \\
& +2 a b \operatorname{Im}\left(r_{p} r_{s}^{*}\right) \cos \alpha \sin \alpha .
\end{aligned}
$$

The polarization extinction ratio is then simply given by $1 / I$. A practical check for $p(s)$ polarized light, namely, for $\beta=0(\pi / 2)$ and the corresponding cross-polarization $\alpha=\pi / 2(0)$ leads to the expected finite polarization leakage $I=b^{2}\left|r_{p / s}\right|^{2}$. For a hypothetical perfect mirror, $r_{p}=1$ and $r_{s}=-1$, making it in this idealized case $I=b^{2}$. Because the reflecting surface has real and imaginary components for $r_{s}$ and $r_{p}$, Eq. (6) shows that for a "sufficiently small value" of $b^{2}$ we can always find a unique choice of angles $\alpha$ and $\beta$ that leads to $I=0$, canceling this way the undesired leakage. This result is always true under condition of total internal reflection, which is the case of a metallic mirror in the visible and infrared range and for a typical cube beam splitter. A sufficiently small value of depolarization to obtain perfect cancellation means in the context of our work typically $b^{2}<6 \times 10^{-3}$ when using a silver mirror as we derive later in the text. The reflecting test surface in combination with the polarizer rotation acts to interfere destructively with the residual rotation-invariant lossless polarization leakage inherent to even the best commercial linear polarizers. Conversely, for a purely dielectric surface such as glass (i.e., BK7) reflecting from the air side, for which $r_{p}$ and $r_{s}$ are both real, no full polarization leakage cancellation is possible. For comparison, we also test our model with a purely dielectric BK7 glass surface with reflectivity from the air side near cross-polarization for the $p$ polarization. The results are shown in Fig. 5(c). In this configuration, as expected from the model, indeed there is no shift $\alpha-\alpha_{0}$ between the condition of maximum cross-polarization for the dielectric and the reference measurement. As expected, there is no effect of cancellation of the polarization leakage. Here, the improved polarization from $10^{5}$ to $10^{6}$ seems to come only from the fact that in $p$ polarization at $45^{\circ}$ the glass surface has a polarizing effect.

To get a better feel for the relevant parameters at work in cancelling almost perfectly the polarization leakage, we use the convenient complex form $r_{p}=\rho_{p} \exp \left(i \varphi_{p}\right)$ and $r_{s}=\rho_{s} \exp \left(i \varphi_{s}\right)$. Consider high-reflectivity mirrors for which $\rho_{p} \approx \rho_{s} \approx 1$. Solving Eq. (4) for field cancellation leads to the first order in $|b| \ll 1$ to a set of two equations $\cos (\alpha-\beta)=-(b / a) \tan \Delta \sin (\alpha+\beta)$ and $\cos (\alpha+\beta)=$ $-(b / a) \cot \Delta \sin (\alpha-\beta)$, where $\Delta=\left(\varphi_{p}-\varphi_{s}\right) / 2$. This way, both $\alpha$ and $\beta$ can be analytically calculated. In the particular case of dielectrics reflecting from the air side for which $\Delta=\pi / 2$, we see already that there are no solutions. Instead, we need the condition $\Delta \neq \pi / 2$, which is always 
verified in condition of total internal reflection. For pure silver at $\lambda=905 \mathrm{~nm}, \varphi_{p}-\varphi_{s}=192.52^{\circ}$, implying $\tan \Delta=-9.12$, which, in turn, shows that for this particular case a solution $I=0$ exists for polarizers with leakage levels $b^{2}<0.012$. One more step is required to make use of these equations toward interpreting our results, because we do not find any easy way to measure independently an absolute value $\alpha$ and $\beta$ to the precision required for our measurements. As explained in the previous section, the value we can measure experimentally with the required accuracy is only the shift $\alpha-\alpha_{0}$. In the reference measurement with the analyzer placed directly after the polarizer, we assume that the cross-polarization condition $\alpha_{0}-\beta=\pi / 2$ holds. For the test experiment, the equation $\cos (\alpha-\beta)=-b \tan \Delta$ is developed in the limit of small leakage $|b \tan \Delta| \ll 1$, so that we get $\alpha-\beta=\pi / 2 \pm b \tan \Delta$ for the near $p$ and $s$ conditions, respectively. This result shows that the correction to the analyzer is simply $\alpha-\alpha_{0}= \pm b \tan \Delta$, corresponding to $\alpha-\alpha_{0}=+0.898^{\circ}$ with a measured leakage $1 / b^{2}=8.3 \times 10^{4}$ and $-0.977^{\circ}$ with $1 / b^{2}=9.6 \times 10^{4}$ for the $p$ and $s$ state, respectively, in the case of the measurement in Fig. 1(b), for a protected silver mirror. Given the measured leakage limiting the nominal extinction at $1 / b^{2}=(1.5 \pm 0.5) \times 10^{5}$, we determine $b=-(2.7 \pm 0.5) \times 10^{-3}$, which, in turn, allows determining $\Delta=99.7^{\circ} \pm 1.7^{\circ}$, a value to be compared to the value for pure silver of $\Delta=96.27^{\circ}$ [38]. We perform an independent measurement of $\Delta$ and find $\Delta=98.3^{\circ}$, which is consistent with our present measurement. Such a value indicates that for our mirror the measured effective $\epsilon_{1}=-23$ instead of -41 for pure silver. This important difference is due to the protective $\mathrm{SiO}_{2}$ layer of about $100 \mathrm{~nm}$ deposited on the silver mirror we use. This simple novel method shows that we can conveniently measure the phase shift $\varphi_{p}-\varphi_{s}$ between $p$ and $s$ polarization after reflection for metals. For a metallic mirror, the phase $\tan \Delta$ is, in fact, related to the angle of incidence $\theta_{i}$, the negative real part of the metal dielectric function $\epsilon_{1}$, and the dielectric function of the protective layer $\epsilon_{\text {back }}$ through the following equation: $\tan \Delta \cong-\sqrt{-\epsilon_{1} / \epsilon_{\text {back }}+\sin ^{2} \theta_{i}} /\left(\sin \theta_{i} \tan \theta_{i}\right)$.

Our measurements performed using a high-reflectivity Bragg mirror show larger shifts $\alpha-\alpha_{0}=\mp 2.2^{\circ}$ leading to a phase shift $\varphi_{p}-\varphi_{s}=189.2^{\circ}$ between the $p$ - and $s$-reflected components.

The full measurements shown in Fig. 1(c) are fitted using Eq. (6), accounting convincingly for the cross-polarization extinction amplification and the slight polarizer and analyzer rotation shift required to reach it. In the limit $\rho_{s} \approx \rho_{p}$, we calculate that the polarization leakage should be sufficiently small to allow perfect extinction when the condition $b^{2}<1 /\left(1+\tan ^{2}(\Delta)\right)$ is verified. For our experimental case, this condition corresponds to $b^{2}<$ $2.1 \times 10^{-2}$ and for a pure silver to $b^{2}<1.19 \times 10^{-2}$. Such values are, in fact, relatively large and, thus, allow realistically achieving polarization leakage cancellation for most standard commercial polarizers.

A last practical aspect to address is the wavelength dependency of this effect. For a highly reflecting mirror, the wavelength dependency is to be found in the phase difference $\Delta(\lambda)$. As a result, the correction to the analyzer angle $\alpha(\lambda)-\alpha_{0}= \pm b \tan \Delta(\lambda)$ calculated for reaching maximum extinction is also a function of the wavelength. Hence, we see that the polarizer angle $\alpha$ of maximum extinction shifts as a function of wavelength as $\partial \alpha / \partial \lambda=$ $\pm b(\partial \Delta / \partial \lambda) / \cos ^{2} \Delta$, which can easily be evaluated using the formulas of the Fresnel coefficient and the corresponding dielectric constant of the mirror-relevant material. For a perfect silver mirror and a polarization leakage of $10^{5}$, we evaluate a chromaticity rate of $\partial \alpha / \partial \lambda=0.0019^{\circ} / \mathrm{nm}$ for a wavelength around $\lambda=905 \mathrm{~nm}$. In this particular example, keeping the analyzer angle at a value of maximum extinction for $905 \mathrm{~nm}$, the wavelength could be shifted by up to $\pm 10 \mathrm{~nm}$ and still keep the extinction up to a level $>10^{7}$.

At this point, we could conclude the paper here, as we are able to explain convincingly all the features of the enhanced polarization extinction. Our analysis, however, misses so far a crucial point, namely, the experimental fact that the leakage cancellation was measurable only in a confocal arrangement, a point that is elucidated in the next sections. More specifically, the analysis we conduct leading to the main result in Eq. (6) so far is done purely for a plane wave for which the Jones matrix formalism is valid. In reality, however, the finite size of the collimated Gaussian laser beam imposes a finite angular wave distribution around the angle of incidence on the mirror [39]. The Fresnel coefficient $r_{p}$ and $r_{s}$ becomes then a function of the angular distribution [39]. This function, as we see, leads to significant geometrical depolarization effects in the form of new optical modes limiting the total extinction to the $10^{4}$ range. We see that a confocal arrangement filters away the depolarization modes and that the result of this section turns out to be fortuitously usable.

\section{MODAL TRANSFORMATION OF A REFLECTED POLARIZED GAUSSIAN BEAM}

To find the origin of the unexpectedly high polarization rejection ratio $>10^{8}$, we map the detected intensity by scanning the spatial position of the collecting fiber in the focal plane of the focusing objective.

In the absence of a reflecting surface between the analyzer and polarizer in cross-polarization, namely, the reference configuration in Fig. 1(a), the measurements in Figs. 2(a) and 2(b) (upper row) show a pure $\mathrm{TEM}_{00}$ Gaussian mode field attenuated by $8.3 \times 10^{4}$ and $9.6 \times 10^{4}$ for a $p$ - and $s$-polarized beam, respectively. This level is expected for the polarizer leakage specifications. In contrast, when we place the analyzer after the 


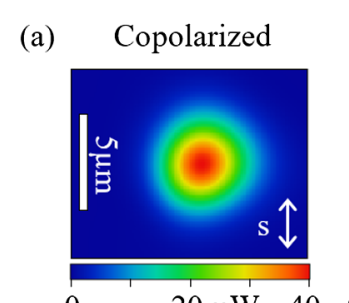

0

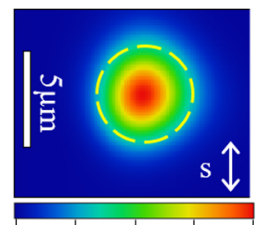

0

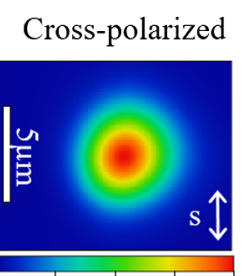

0

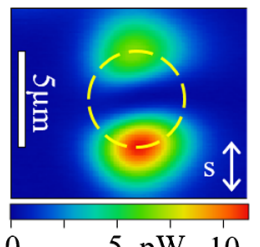

Extinction ratio

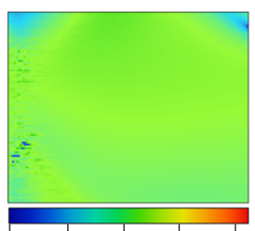

$\begin{array}{lllll}0 & 10^{2} & 10^{4} & 10^{6} & 10^{8}\end{array}$

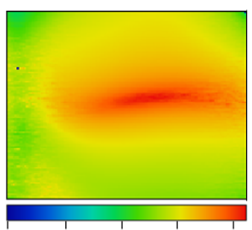

$\begin{array}{lllll}0 & 10^{2} & 10^{4} & 10^{6} & 10^{8}\end{array}$ (b)

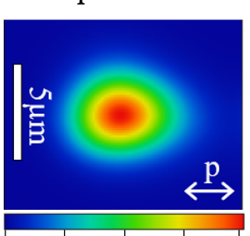

0

$40 \mu \mathrm{W} 80$

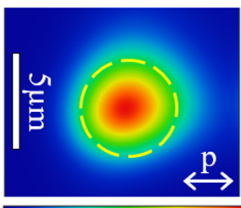

$25 \mu \mathrm{W} 50$

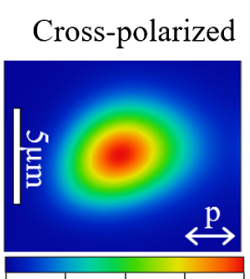

0

$0.5 \mathrm{nW} \quad 1$

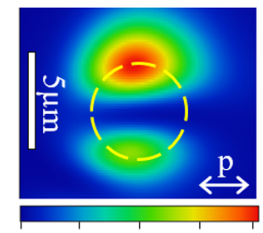

$35 \mathrm{pW} 70$
Extinction ratio

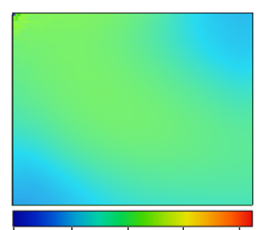

$\begin{array}{llllllll}0 & 10^{2} & 10^{4} & 10^{6} & 10^{8}\end{array}$

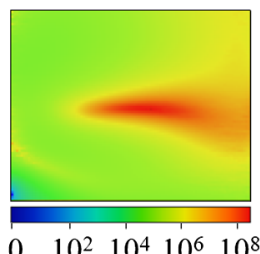

FIG. 2. Confocal mapping of $s$ (a) and $p$ (b) laser beams in co- and cross-polarization using a scanning mirror (9). The upper and lower rows of the figures are measurements with the analyzer placed before [Fig. 1(a)] and after [Fig. 1(b)] the test reflecting surface, respectively, in $s$ polarization and $p$ polarization [(a) and (b), respectively]. We plot the extinction ratio map by dividing, pixel per pixel, the copolarized with the cross-polarized data. In cross-polarization, a modal splitting along $\vec{e}_{y}$ above and below the plane of incidence is observed for both the $s$ and $p$ polarization. The dotted line circle is the $1 / e$ intensity level of the Gaussian distribution resulting from the confocal convolution between the focused spot and the collecting fiber mode. The location of the maxima of the modal splitting lies exactly on that circle. The diameter of this circle gives also the nonconvoluted focal spot waist diameter at $1 / e^{2}$ focused on the collecting fiber end. The vertical white bar represents $5 \mu \mathrm{m}$.

reflecting test surface, the measurements show that the mode splits into two lobes distributed along $\vec{e}_{y}$ and located above and below the reflectivity plane. In this crosspolarized configuration, we find an intensity "hole" at the location of the optical fiber center. There, the intensity extinction is slightly higher than $10^{8}$, a factor of 100 away from our actual setup sensing limit. We occasionally reach $10^{10}$ records in which the dark noise of the detector is, in fact, the limiting factor. We experimentally verify the stability of this effect over tens of hours for $p$ and $s$ linearly polarized incident light. We observe qualitatively the same effects for incidence angles of $\theta_{i}$ at $9^{\circ}, 22^{\circ}, 25^{\circ}$, $30^{\circ}$, and $68^{\circ}$. We observe qualitatively the same behavior for different type of polarizers such as a crystal polarizer (Glan-Taylor) and nanoparticle thin film linear polarizers, for different mirrors such as silver, gold, aluminum, dielectric Bragg reflectors, and nonpolarizing beam-splitter cubes, attesting to the robustness of this effect.

To get a feel for the measured modal transformation for $p$ and $s$, we measure and show in Figs. 3 and 4 the evolution of the confocal light intensity maps for different analyzer rotation angles variation $\delta \alpha$ around the symmetrically split mode. Figures 3(d) and 4(d) show quantitatively for $p$ and $s$ polarizations the measured positions of beam-peak shifts along $\vec{e}_{y}$ and splitting above and below the plane of incidence as a function of $\delta \alpha$. We observe a very similar behavior for beam-splitter cubes typically used in the resonant fluorescence setup such as in Refs. [17,18,24,31], with the difference, however, that the equivalent figure looks instead mirrored with respect to the axis $\Delta y=0$. In all cases, such split-lobe intensity distribution is very reminiscent of a $\mathrm{TEM}_{01}$ HermitGaussian mode.

We reach a record level of $10^{10}$ for which the limiting factor is the dark noise of our detector. The challenge in such an experiment is to have a polarization rotator that enables stepping with small enough rotation angles. At this point, we need to find out why (i) the confocal arrangement enables the dramatic extinction enhancement as seen in Fig. 1(c) and (ii) why the beam shifts and splits at cross-

(a)

(b)
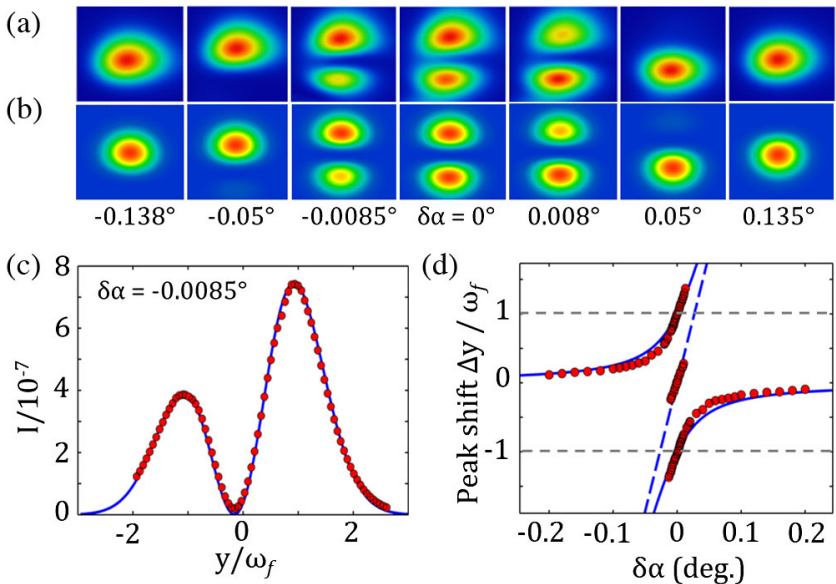

FIG. 3. p-polarized beam reflected off a silver mirror. Measured (a) and simulated (b) evolution of the modal confocal imaging mapping through maximum extinction (c) for different analyzer angles $\delta \alpha$ as explained in the text. In (d), the positions of the intensity extrema are shown in units of beam waist $\omega_{f}$ at focus. 
(a)

(b)

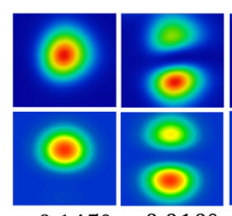

$-0.145^{\circ}-0.010^{\circ}$

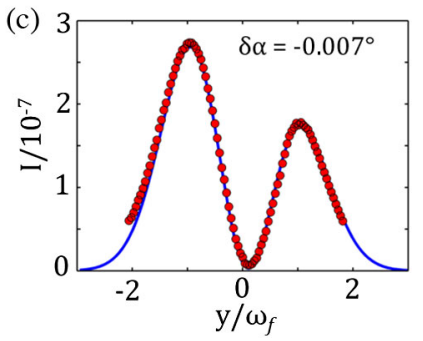

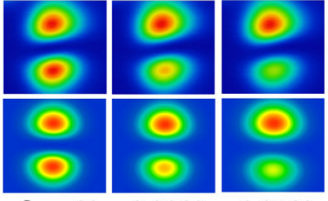

$0.012^{\circ} \quad 0.148^{\circ}$

(d)

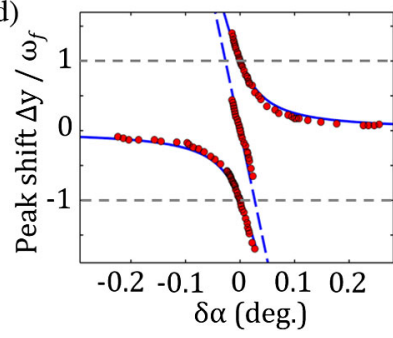

FIG. 4. $s$-polarized beam reflected off a silver mirror. Measured (a) and simulated (b) evolution of the modal confocal imaging mapping through maximum extinction (c) for different analyzer angles $\delta \alpha$ as explained in the text. In (d), the positions of the intensity extrema are shown in units of beam waist $\omega_{f}$ at focus.

polarization in Fig. 2 (lower row) and always above and below the plane of incidence.

To answer these questions, we need to first model the spatial field $\vec{E}_{f x, y}$ at the focal plane of the focusing lens just before the collecting single-mode optical fiber and then use the collecting fiber as the confocal Gaussian filter function porting the light to the detector. The finite-size beam before the mirror results from a Gaussian-weighted superposition of plane waves propagating along an angular distribution $\vec{k} / k_{0}=u \vec{p}+v \vec{s}+w \vec{k}_{0} / k_{0}$ very narrowly centered around $\overrightarrow{k_{0}}$ the wave vector along the optical axis with $k_{0}=2 \pi / \lambda$. In the paraxial approximation, $u$ and $v$ are both $\ll 1$, so they represent the angular spread of the collimated beam. The focusing lens transforms each plane-wave component $\overrightarrow{\mathcal{E}}_{u, v}$ of the angular distribution into a field $\vec{E}_{f x, y}$ in the focal plane; hence, the beam reaching the focal plane at distance $f$ results from a coherent superposition of all such focused components. The spatial distribution of the field at a point located at spatial coordinates $(p, s, z)$ after the mirror is given as

$\vec{E}(p, s, z)=\iint_{-\infty}^{+\infty} \overrightarrow{\mathcal{E}}\left(k_{p}, k_{s}, k_{z}\right) e^{+i k_{p} p+i k_{s} s+i k_{z} z} d k_{p} d k_{s}$.

The next step is to obtain the angular distribution $\overrightarrow{\mathcal{E}}_{u, v}$. Such a problem is modeled for a Gaussian field distribution in Ref. [40] by Aiello and Woerdman. We derive here a simplified version conveniently describing the essential physics needed to model our observations. We begin with the field just before the polarizer, for which we assume a linearly polarized Gaussian-field normalized angular distribution $\overrightarrow{\mathcal{E}}_{0 u, v}$ :

$$
\overrightarrow{\mathcal{E}}_{0 u v}=\frac{\omega_{0}^{2}}{4 \pi} E_{0} \exp -\frac{u^{2}+v^{2}}{\theta_{0}^{2}}\left[\begin{array}{c}
\cos \beta \\
\sin \beta
\end{array}\right] .
$$

The mode divergence $\theta_{0}=2 /\left(k_{0} \omega_{0}\right)=\omega_{0} / l$ results from the finite size of the collimated laser beam with beam radius $\omega_{0} \equiv 3 \mathrm{~mm}$, and the Rayleigh range $l=k_{0} \omega_{0}^{2} / 2$ is $31.24 \mathrm{~m}$, a value much larger than the size of our experimental setup, allowing us to ignore the role of beam propagation up to the focusing lens. Here, the distance between the mirror of interest (8) and the focusing objective is typically $0.3 \mathrm{~m}$. With this convention, a $p$ - $(s)$ - polarized light is obtained at $\beta=0(\pi / 2)$. When the beam reflects off the test surface, each plane-wave component acquires an angle-dependent Fresnel reflection coefficient $r_{p, u v}$ and $r_{s, u v}$ that are functions not only of $\theta_{i}$ but also of $u$ and $v$ [39]. Consequently, for each plane-wave component, we choose a coordinate system $\vec{e}_{p}, \vec{e}_{s}, \vec{k} / k_{0}$ that defines a local incidence plane for that wave. The longitudinal basis vector is $\vec{k} / k_{0}$, and the transverse ones are $\vec{e}_{s}=\vec{k} / k_{0} \times \vec{e}_{z}$ and $\vec{e}_{p}=\left(\vec{k} / k_{0} \times \vec{e}_{z}\right) \times\left(\vec{k} / k_{0}\right)$ in the $s$ and $p$ planes, respectively. To obtain the reflectivity of the mirror for each plane wave, we determine first the weights of $p$ - and $s$-field components, given by the weighted projections $r_{p, u v}\left(\vec{e}_{p} \cdot \overrightarrow{\mathcal{E}}_{0 u v}\right)$ and $r_{s, u v}\left(\vec{e}_{s} \cdot \overrightarrow{\mathcal{E}}_{0 u v}\right)$. We determine then the resulting reflected field transverse field along the corresponding reflected basis $\vec{e}_{s, R}=\vec{k}_{R} / k_{0} \times \vec{e}_{z}$ and $\vec{e}_{p, R}=\left(\vec{k}_{R} / k_{0} \times \vec{e}_{z}\right) \times\left(\vec{k}_{R} / k_{0}\right)$ such that $\overrightarrow{\mathcal{E}}_{u v}=$ $r_{p, u v}\left(\vec{e}_{p} \cdot \overrightarrow{\mathcal{E}}_{0 u v}\right) \vec{e}_{p, R}+r_{s, u v}\left(\vec{e}_{s} \cdot \overrightarrow{\mathcal{E}}_{0 u v}\right) \vec{e}_{s, R}$. Here, $\vec{k}_{R}$ is the mirrored wave vector after reflection. In the paraxial limit, for a beam impinging, the Fresnel coefficients are developed to the first order in $u$ around $\theta_{i}$ and $v$ around 0 , giving $r_{p, u v}=r_{p}+u \partial r_{p} / \partial \theta_{i}$ and $r_{s, u v}=r_{s}+u \partial r_{s} / \partial \theta_{i}$. The first-order derivatives $\partial r_{p / s u v} / \partial v$ in the $s$ plane vanish for both $r_{p}$ and $r_{s}$, leaving just derivatives $r_{p}^{\prime}=\partial r_{p} / \partial \theta_{i}$ and $r_{s}^{\prime}=\partial r_{s} / \partial \theta_{i}$. We calculate the components of the incoming and reflected basis vectors $\vec{e}_{p}, \vec{e}_{s}, \vec{k} / k_{0}$ and $\vec{e}_{p, R}$, $\vec{e}_{s, R}, \vec{k}_{R} / k_{0}$ in the paraxial limit $u, v \ll \theta_{i}$. After a lengthy but straightforward calculation, we obtain the reflected field distribution after the mirror for each angle $u, v$. We express the result conveniently in terms of matrix notation $\vec{E}_{u v}=\overline{\bar{M}}_{u, v} \vec{E}_{0 u v}$, where

$\overline{\bar{M}}_{u, v}=\left[\begin{array}{cc}r_{p} & 0 \\ 0 & r_{s}\end{array}\right]+u\left[\begin{array}{cc}r_{p}^{\prime} & 0 \\ 0 & r_{s}^{\prime}\end{array}\right]+v \frac{r_{p}+r_{s}}{\tan \theta_{i}}\left[\begin{array}{cc}0 & -1 \\ 1 & 0\end{array}\right]$.

Upon inspection of the expression (9) for symmetries, we see now that the reflectivity Jones matrix transforms an impinging perfect Gaussian mode, such as Eq. (8), into the sum of $\mathrm{TEM}_{00}, \mathrm{TEM}_{01}$, and $\mathrm{TEM}_{10}$ Hermit-Gauss modes. The indices for $\mathrm{TEM}_{n m}$ indicate the number of nodes along 
the $\vec{p}$ and $\vec{s}$ direction, respectively. The first term in the right-hand side is the normal test surface reflectivity we use in the first part of this paper. The second term is responsible for generating a TEM 10 mode along $\vec{p}$ in the plane of incidence. This term is, in fact, responsible for the GoosHänchen effect [41], as it has its physical origin in the angular dispersion of the reflectivity terms at $\theta_{i}$. Here, the different plane-wave components acquire slightly different phases upon reflectivity shifting the beam in the plane of incidence. Because this matrix is diagonal, we see that for a perfect $p$ or $s$ polarization the Goos-Hänchen effect does not contribute to depolarization. The third term, the most relevant to this work, is responsible for generating an outof-plane-of-incidence $\mathrm{TEM}_{01}$ mode with two lobes along $\vec{e}_{y}$. This term is the physics responsible for the ImbertFedorov effect $[32,33,36,42]$ known to deviate a reflected light beam above or below the plane of incidence depending on its right-handed or left-handed polarization helicity. The calculation detailed above shows that this term originates purely from geometrical projections in which the gradual phase shift gained by each plane-wave component upon reflection sums to a cross-diagonal matrix that mixes the $p$ - and $s$-phase-shifted reflected plane-wave components. Consequently, this term is responsible for an intrinsic reflectivity-induced depolarization for $p$ and $s$ polarization even when using ideally perfect polarizers. Because of the purely geometrical projection nature of the argumentation, compelling connections between the Imbert-Fedorov effect, Berry's phase, and spin-Hall effect of light are discussed in the literature [36,42]. Because of the direct proportional dependency of this matrix on the angle $v$ and, in particular, its sign, it creates a TEM $_{10}$ mode asymmetric along $\vec{e}_{y}$, adding (subtracting) the field to (from) the symmetric main mode, displacing this way its weight above or below the plane of incidence depending on its helicity. This asymmetry can be easily verified using a circular polarization version of Eq. (8) with the Jones matrix equation (9). From this simple derivation, it is worth appreciating that in the paraxial approximation Eq. (9) expresses both Goos-Hänchen and Imbert-Fedorov effects in an elegant and compact way. At this point, we can see from a symmetry argument that our confocal arrangement enhances cross-polarization extinction.

In the following step, we express the field distribution transmitted through the polarizer, the mirror, and analyzer at the back aperture of the focusing lens $\overrightarrow{\mathcal{E}}_{u v}=$ $\overline{\bar{A}}(\alpha) \overline{\bar{M}}_{u, v} \overline{\bar{P}}(\beta) \overrightarrow{\mathcal{E}}_{0 u v}$ used in the Fourier transform equation (7). Before providing the general solution, we get first a feel for the physical parameters governing the Imbert-Fedorov cross-polarized mode. We consider the special case of a $p$ - or $s$-polarized light impinging on the mirror and subsequently analyzed in cross-polarization configuration. Here, only the third matrix on the rhs of Eq. (9) is relevant, and all other terms cancel.
The essential physical parameter that governs the ImbertFedorov mode field intensity is the sum $r_{p}+r_{s}$ given by the material reflecting properties. To get a more physical insight, we use the representation $r_{p}=\rho_{p} \exp \left(i \varphi_{p}\right)$ and $r_{s}=\rho_{s} \exp \left(i \varphi_{s}\right)$ that can be conveniently symmetrized using $\rho_{s}=\rho+\delta \rho / 2$ and $\rho_{p}=\rho-\delta \rho / 2$ for the reflectivity and $\Delta=\left(\varphi_{p}-\varphi_{s}\right) / 2$ the phase difference. This way, we obtain

$$
r_{p}+r_{s}=2 \rho \cos \Delta+i \delta \rho \sin \Delta
$$

to within a constant proportional phase term $\exp i\left(\varphi_{p}+\right.$ $\left.\varphi_{s}\right) / 2$ identical for all modes of Eq. (9). We now see that the difference $\varphi_{p}-\varphi_{s}$ governs the intensity and the phase of the Imbert-Fedorov mode. For instance, in the case of the air side reflectivity off a perfect dielectric, we have $\varphi_{p}-\varphi_{s}=\pi$, and, hence, $r_{p}+r_{s}=i \delta \rho$, so the ImbertFedorov mode field intensity is directly proportional to the pure differential reflectivity between the $p$ and $s$ waves. In contrast, for dielectrics under total internal reflectivity and for metals, we have $\delta \rho \approx 0$ and $\rho \approx 1$ so that $r_{p}+r_{s}=2 \cos \Delta$. In this case, the strength of the depolarizing mode is fully governed by the phase difference $\varphi_{p}-\varphi_{s}$. We conclude that mapping the Imbert-Fedorov mode fields in a confocal microscopy setup provides a direct and sensitive access to the differential reflectivity amplitude and phases of a reflecting surface. To move forward with our analysis on the more general case, we perform the Fourier optics transformation equation (7). The result is that the field image at the entrance of the lens for the test experiment with the mirror placed between the polarizers is $\vec{E}(p, s, z)=\overline{\bar{A}}(\alpha) \overline{\bar{M}}_{p, s, z} \overline{\bar{P}}(\beta) \vec{E}_{0}(p, s, z)$, where the effective reflectivity Jones matrix is given by

$$
\begin{aligned}
\overline{\bar{M}}_{p, s, z}= & {\left[\begin{array}{cc}
r_{p} & 0 \\
0 & r_{s}
\end{array}\right]+\frac{i p}{l+i z}\left[\begin{array}{cc}
r_{p}^{\prime} & 0 \\
0 & r_{s}^{\prime}
\end{array}\right] } \\
& +\frac{i s}{l+i z} \frac{r_{p}+r_{s}}{\tan \theta_{i}}\left[\begin{array}{cc}
0 & -1 \\
1 & 0
\end{array}\right] .
\end{aligned}
$$

The spatial distribution of the field $\vec{E}(p, s, z)$ results from the Fourier transform of the unperturbed linearly polarized laser field angular distribution of Eq. (8) into a spatial normalized distribution such that

$$
\vec{E}_{0}(p, s, z)=e^{i k_{0} z} \exp -\frac{p^{2}+s^{2}}{\omega_{0}^{2}(1+i z / l)}\left[\begin{array}{c}
\cos \beta \\
\sin \beta
\end{array}\right] .
$$

The resulting reflected field distribution arriving at the lens turns out to be the one discovered in a different context in the insightful and pioneering work of Aiello and Woerdman [43]. In their work, the authors provide, within a paraxial approximation, a complete analytical solution for the field distribution of a single-mode Gaussian 
beam reflected off a mirror. The next step is to perform the lens transformation to obtain the field in the focal plane $x, y[44]$ :

$$
\begin{aligned}
& \vec{E}(x, y, f) \\
& \quad=-i \frac{e^{2 i\left[\left(x^{2}+y^{2}\right) / \omega_{0} \omega_{f}\right]}}{\pi \omega_{0} \omega_{f}} \iint_{-\infty}^{+\infty} \vec{E}(p, s, f) e^{-2 i\left[(x p+y s) / \omega_{0} \omega_{f}\right]} d p d s,
\end{aligned}
$$

where the focused spot waist radius $\omega_{f}=\lambda f / \pi \omega_{0} \simeq$ $2.5 \mu \mathrm{m}$ is the fiber Gaussian mode size and $l_{f}=k_{0} \omega_{f}^{2} / 2$ its corresponding Rayleigh range, in our case $21.7 \mu \mathrm{m}$. It is useful to note that $l_{f}\left(\omega_{0} / \omega_{f}\right)=f$. We calculate the resulting field spatial distribution in the focal plane:

$$
\begin{aligned}
\vec{E}(x, y, f)= & \left(-i+\frac{z}{l}\right) e^{i k_{0} z} e^{i \frac{2\left(x^{2}+y^{2}\right)}{\omega_{0} \omega_{f}}} e^{-\frac{\left(x^{2}+y^{2}\right)}{\omega_{f}^{2}(1+i z / l)}} \\
& \times \frac{\omega_{0}}{\omega_{f}}\left\{\overline{\bar{M}}_{0}+\frac{x}{f} \overline{\bar{M}}_{G H}+\frac{y}{f} \overline{\bar{M}}_{I F}\right\} \vec{E}_{0} .
\end{aligned}
$$

The last matrix term gives the cross-polarized field component which is an antisymmetric function of $y$ with two lobes with opposite phase and maxima located at $y=$ $\pm \omega_{f} / \sqrt{2}$ above and below the plane of incidence. The essential finding from our work is that the confocal arrangement transforms the collimated beam waist $\omega_{0}$ and Rayleigh length $l+i z$ of the Aiello and Woerdman [43] field distribution at the mirror plane, into $\omega_{f}$ and if in our case. Aiello and Woerdman [43] show that it is the finite size of the beam at the reflecting surface that is responsible for the additional field terms that affect the initial Gaussian mode. This result amounts to a sizable amplification of the weak mode intensity in proportion to $\omega_{0} / \omega_{f}$. From our work, it is becoming now clear here that a confocal arrangement provides a valuable advantage to explore experimentally the cross-polarization geometry with sufficient sensitivity and a very fine spatial resolution. In particular, high-extinction cross-polarization extinction is kin to the "weak measurement procedure" of Aharonov et al. $[45,46]$, which we extend here to a confocal arrangement enabling the added benefit of spatial resolution. Recent literature [36] provides an interpretation for the depolarization as resulting from an effective spin-orbit interaction of light occurring at the mirror surface manifesting itself in the form of a spin-Hall effect of light [47]. In this work, we restrict ourselves to a purely modal interpretation and leave the discussion concerning spin orbit aside.

\section{EFFECT OF CONFOCAL SPATIAL FILTERING}

The one final point we need to address to get a full quantitative interpretation of our experiment is to address the effect of the confocal filter function of the collecting fiber. The single-mode fiber collects and ports the field to the photodetector; it does this collection, however, by acting as a Gaussian spatial filter. For our symmetric setup shown in Fig. 1, we illuminate and collect light with a single-mode fiber of identical mode size and with identical collimating and focusing lenses. The spatial filtering is a convolution between the field at the focal plane and the fiber Gaussian mode amounting to an integrated field $\pi \omega_{f}^{2} \vec{E}_{D}$ at location $x_{0}, y_{0}$ of the fiber with respect to the optical axis. The power measured at the other end of the fiber is $\pi \omega_{f}^{2} \epsilon_{0} c \vec{E}_{D} \vec{E}_{D}^{+}$. The results of the calculation for the field are the following. First, we get the mapping of the reference field without the use of the polarizers and the reflecting surface as measured by the detector:

$$
\vec{E}_{D 0,0}=\left(-i+\frac{z}{l}\right) \frac{\omega_{0}}{\omega_{f}} E_{0} e^{i k_{0} z} \exp -\gamma \frac{x_{0}^{2}+y_{0}^{2}}{2 \omega_{f}^{2}}\left[\begin{array}{c}
\cos \beta \\
\sin \beta
\end{array}\right],
$$

where $\gamma=\left(1+2 \eta^{2}\right) /(1+i \eta)$ and $\eta=\omega_{f} / \omega_{0}+z / 2 l$. We note that the beam waist at focus appears now to be broadened by a factor of $\sqrt{2}$ when comparing with the distribution of Eq. (13). Second, we find that the confocal filtering by convolution with a Gaussian mode leads to a modified effective Jones matrix for the reflecting surface acting on the field as seen from the detector:

$\overline{\bar{M}}_{D x_{0}, y_{0}}=\left[\begin{array}{cc}r_{p} & 0 \\ 0 & r_{s}\end{array}\right]+\frac{x_{0}}{2 \zeta}\left[\begin{array}{cc}r_{p}^{\prime} & 0 \\ 0 & r_{s}^{\prime}\end{array}\right]+\frac{y_{0}}{2 \zeta} \frac{r_{p}+r_{s}}{\tan \theta_{i}}\left[\begin{array}{cc}0 & -1 \\ 1 & 0\end{array}\right]$,

where $\zeta=f(1-i \eta)$ is a complex-valued length that we use as a fitting parameter when comparing our experimental data. With the confocal filtering, the Goos-Hänchen and the Imbert-Fedorov field terms (i.e., the second and third terms of the rhs in the equation) are halved when compared to Eq. (14). The Jones matrix related to the polarizers and polarization leakage remains unchanged. With this last correction, we have now all the equations required in order to simulate the modal transformation induced by a reflecting surface acting on a polarized Gaussian beam and for any arbitrary polarization and polarization leakage level. Finally, the full scanning confocal mapping of the detected field is given by the analytical form

$$
\vec{E}_{D}\left(x_{0}, y_{0}\right)=\overline{\bar{A}}(\alpha) \overline{\bar{M}}_{D x_{0}, y_{0}} \overline{\bar{P}}(\beta) \vec{E}_{D 0,0}
$$


The first important result we are getting from Eq. (17) and the mirror matrix term in Eq. (16) is when the location of the fiber center and the focal spot axis coincide, namely, for $x_{0}=0$ and $y_{0}=0$. In this case, the result is the same as found in the simplified plane wave analysis in Sec. III, and the equation of polarization cancellation given in Eq. (6) holds fortuitously. This result is the case because the filtering function of the confocal arrangement eliminates the higher depolarizing modes. Without the confocal filtering, the normalized integrated total intensity in cross-polarization detected in wide field imaging of the focused point, or collected with a wide core multimode fiber, is estimated by summing the measured light intensity collected in the cross-polarization mode such as shown in Fig. 2 and normalizing to the integrated intensity in the copolarized configuration. This estimation gives for our silver mirror a best extinction possible in the range of $10^{6}$, namely, for the best case for which we are using the polarization leakage compensation approach described earlier.

This result demonstrates the key role of the confocal arrangement for the giant polarization extinction reached in the state of the art resonance fluorescence measurements $[17,18,24,31]$.

The second significant result is illustrated, applying Eq. (17) on a purely $p$ - or $s$-polarized beam measured in cross-polarization, by mapping the focused spot position $\left(x_{0}, y_{0}\right)$ across the single-mode fiber end. The result is an intensity map displaying two lobe maxima located at the fiber location at $x_{0 \max }=0$ and $y_{0 \max }= \pm \omega_{f}$ above and below the plane of incidence. This result is in qualitative agreement with our measurements as seen in Fig. 2 for silver. We confirm these findings for Bragg mirrors and thin-film-based beam-splitter cubes. The third significant result is depicted in Figs. 3(d) and 4(d), showing the evolution near cross-polarization of the position of the reflected maxima of intensity as well as the location of the intensity node as a function of the analyzer angle. Here, the best fit to the data obtained with Eq. (17) imposes a purely imaginary value to $\zeta$ about $i 600 \mu \mathrm{m}$. Failure to use an imaginary value for $\zeta$ leads to a radically different calculated dependency of the intensity extrema near crosspolarization for Figs. 3(d) and 4(d). The predicted evolution would be instead the one seen in Fig. 5(d) for a dielectric illuminated from the air side. This result came as a complete surprise to us, and we have no explanation for why we need to use a purely imaginary $\zeta$ to fit all our data. The other surprise, aside from the imaginary value aspect, is that the absolute value of $\zeta$ is about 40 times shorter than the focal length $f$ that would be normally close to the theoretical value of $\zeta$ to be normally used to interpret our experiment. We conduct a similar measurement on a BK7 glass surface illuminated from air. The measured and fit data are shown in Fig. 5(d). Here as well, the value of $\zeta$ to get the best fit requires a purely imaginary value consistent (a)

(b)

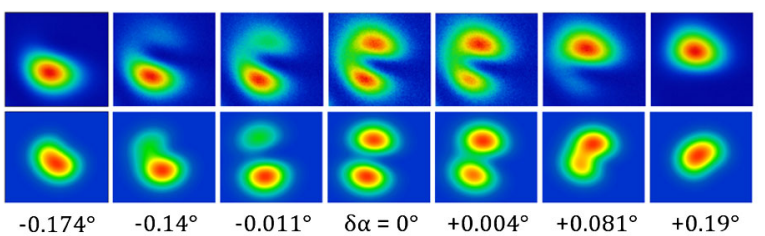

(c)
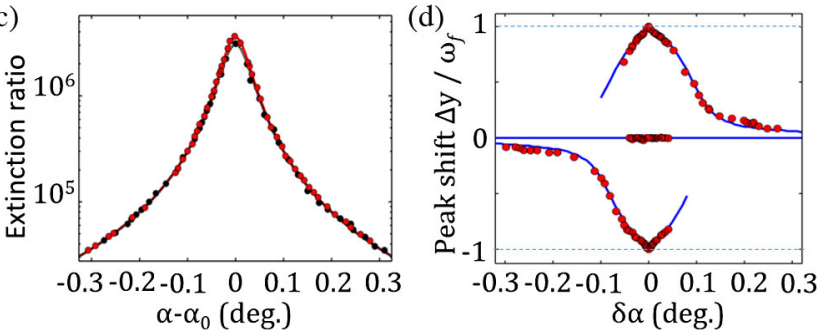

FIG. 5. Measured (a) and simulated (b) evolution of the modal mapping through maximum extinction for reflectivity from the air side off a glass surface (BK7) for $p$ polarization. (c) Red symbols: extinction ratio for different analyzer angle $\alpha$ shifts as explained in the text. Black symbols: reference measurement with the analyzer placed just after the polarizers. The positions of the intensity extrema are shown in units of beam waist $\omega_{f}$ at focus.

with the value used for Figs. 3(d) and 4(d). Numerous other measurements performed in the circular polarization basis as well as in a linear polarization basis $45^{\circ}$ tilted from $p$ and $s$ confirm the necessity to use an imaginary value for the length parameter $\zeta$, showing that our understanding is still incomplete. We believe that we are still missing a full quantitative understanding in the way the receiving fiber filters non-Gaussian modes. The use of an imaginary value of $\zeta$ maps closely the evolution of the mode transformation near the cross-polarization condition for both normal and total internal reflection. In our experiment, the material parameter of the high-reflectivity surface that governs most of the effects we observe is the phase difference $\varphi_{p}-\varphi_{s}$. In particular, for high-reflectivity materials and total internal reflection, the intensity of the lobe maxima at crosspolarization is obtained from Eq. (16) at fiber location $\left(x_{0}, y_{0}\right)=\left(0, \pm \omega_{f}\right)$ :

$$
\left|\vec{E}_{D}\left(0, \pm \omega_{f}\right) / \vec{E}_{D 0,0}\right|^{2}=\frac{1}{4 e} \frac{\left|r_{p}+r_{s}\right|^{2}}{\tan ^{2} \theta_{i}}\left(\frac{\omega_{f}}{\zeta}\right)^{2}
$$

In particular, for high-reflectivity materials, from Eq. (11), we have $\left|r_{p}+r_{s}\right|^{2} / 4=\cos ^{2} \Delta$. In our experiment, we determine earlier that for the protected silver surface $\Delta=100^{\circ}$, so using the value of $600 \mu \mathrm{m}$ we fit for the absolute value of $\zeta$, and the lobe intensity should be about $2 \times 10^{-7}$, which is in the range of our measured values. We measure typical lobe maxima in the range of $0.3-1.4 \times 10^{-6}$. For a direct comparison, we test our model with a purely dielectric BK7 glass surface with reflectivity from the air side near cross-polarization for the $p$ polarization. The results are shown in Fig. 5. In this 
configuration, as expected from the model discussed in Sec. III, indeed there is no shift $\alpha-\alpha_{0}$ between the condition of maximum cross-polarization for the dielectric and the reference measurement. As expected also from Sec. III, there is no effect of cancellation of the polarization leakage. The most remarkable difference is the way the mode splitting evolves upon rotation of the analyzer. The absence of imaginary terms in $r_{p}$ and $r_{s}$ is the reason for this behavior. Here, we see not only the Imbert-Fedorov out-of-plane splitting at cross-polarization but also the appearance of the Goos-Hänchen mode showing a mixing that bends the beam shape along the plane of incidence. Figure 5 shows, in particular, the evolution of the beam splitting near the cross-polarization condition which is completely different from what is seen for metals such as in Figs. 3(d) and 4(d). Because $\operatorname{Im}\left(r_{s} r_{p}^{*}\right)=0$ for the reflectivity from the air side of a dielectric, there is no term linear in $\delta \alpha$ near cross-polarization conditions as also seen from the experiments. For a dielectric, an analytical solution for the location of the lobe intensity maxima for very small analyzer rotation angle $\delta \alpha \ll 1$ near the crosspolarization condition shows the quadratic evolution observed in our measurement.

As a final note, we believe that we are still missing a full quantitative understanding in the way the receiving fiber filters non-Gaussian modes. Indeed, the sensitivity of our setup should have permitted us to detect the higher-term modes $\mathrm{TEM}_{11}$ that have a symmetry $x y$. Such modes originate from the finite-sized waist of the Gaussian beam, making it naturally divergent [48]. In fact, we can use the exact formalism developed above to show that such terms originate also from geometrical projections around the optical axis. This time, the projection is not involving any reflecting surface but just the natural divergence of the beam before the lens, leading to a gradual phase shift gained by each plane-wave component, here again depolarizing naturally the beam. Keeping in mind the convolution imposed by the collecting fiber, we calculate that the expected clove-shaped mode is peaking at the four locations $\left(x_{0} ; y_{0}\right)=\left( \pm \omega_{f} ; \pm \omega_{f}\right)$ with an intensity given by

$$
\left|\vec{E}_{D}\left( \pm \omega_{f}, \pm \omega_{f}\right) / \vec{E}_{D 0,0}\right|^{2}=\frac{1}{4 e^{2}}\left(\frac{\omega_{f}}{2 l_{f}}\right)^{4}
$$

a result corroborated in Ref. [48] and in Ref. [39]. Using this expression for our experiment parameters, the mode peak intensity should be $3.8 \times 10^{-7}$, a value that is well within our sensitivity range. It is a puzzling fact that we do not observe any trace of this $\mathrm{TEM}_{11}$ signal. There is no doubt, however, that this mode is present as measured in Ref. [48]; this difference is why we believe that our understanding of the way the optical fiber is filtering the signal is not complete yet.

\section{CONCLUSIONS}

In conclusion, we have exposed a systematic experimental method based on a confocal microscopy arrangement to obtain a giant enhancement in dark-field crosspolarization extinction and by up to 3 orders of magnitude and possibly beyond. We found that the effect exploits the material properties of a surface or interface under a condition of total internal reflectivity, in particular. Our work provides a good starting point for further research aiming to optimize the performance of the microscope optics in terms of polarization extinction. According to our work, future research should focus on the phase characteristics of reflecting surfaces (such as beam-splitter cubes and mirrors) to cancel depolarization effects instead of merely developing strategies to make very sophisticated, inconvenient, and extremely expensive microscope objectives. Our approach is simple, and the high polarization rejection can be achieved for a targeted wavelength excitation and is based only on polarization properties of light. This basis means it has the potential to open up a broad spectrum of innovative opportunities for applications and new forms of interdisciplinary research including biology [49], plasmonics [50], and optoelectronics [51], where resonant confocal microscopy starts to play an important role.

Future developments in quantum optics based on novel nanostructures demand an enhanced quantum coherence, for instance, quantum entanglement between electronic spin state and photons which can be achieved only using resonant laser spectroscopy. Thus, an extreme cross-polarization extinction coefficient $\left(>10^{8}\right)$ is very much the key to guarantee the optical spin coherence property of single photons. The technique presented here addresses promising quantum optical systems currently explored in a large variety of materials for which Stokes-shifted readout of a coherent few-level system is not possible or inconveniently weak. This was the reason why semiconductor quantum dot research profited very much from this technique and was immediately adapted to single-molecule coherent spectroscopy [52]. In the future, resonance fluorescence could favor the use of fluorophores that have weak intersystem crossing rates. Such molecules are attractive for their much increased photostability but at the cost of a much reduced Stokes shift. Wide-band-gap materials are interesting not only for their deep-level impurities but also for their quantum confinement such as in $\mathrm{GaN}$ and similar systems. Here, there is no Stokes shift to allow for the readout of the fundamental level of the system. The same goes also for quantum confined layered system such as $\mathrm{WSe}_{2}$ as also recently studied by resonance fluorescence by cross-polarization confocal microscopy [12].

\section{ACKNOWLEDGMENTS}

We thank R. Warburton, M. Kroner, L. Novotny, O. Krebs, and C. Schaefermeier for useful discussions. Part of 
this project has received funding from the European Union's Horizon 2020 research and innovation program under the Marie Skłodowska-Curie Grant Agreement No. 721394 ITN 4PHOTON.

[1] I. Aharonovich, D. Englund, and M. Toth, Solid-State Single-Photon Emitters, Nat. Photonics 10, 631 (2016).

[2] A. Högele, M. Kroner, C. Latta, M. Claassen, I. Carusotto, C. Bulutay, and A. Imamoglu, Dynamic Nuclear Spin Polarization in the Resonant Laser Excitation of an InGaAs Quantum Dot, Phys. Rev. Lett. 108, 197403 (2012).

[3] M. Paillard, X. Marie, P. Renucci, T. Amand, A. Jbeli, and J. M. Gerard, Spin Relaxation Quenching in Semiconductor Quantum Dots, Phys. Rev. Lett. 86, 1634 (2001).

[4] A. Högele, S. Seidl, M. Kroner, K. Karrai, R. J. Warburton, B. D. Gerardot, and P. M. Petroff, Voltage-Controlled Optics of a Quantum Dot, Phys. Rev. Lett. 93, 217401 (2004).

[5] M. Atatüre et al., Quantum-Dot Spin-State Preparation with Near-Unity Fidelity, Science 312, 551 (2006).

[6] Y. Arakawa and M. J. Holmes, Progress in Quantum-Dot Single Photon Sources for Quantum Information Technologies: A Broad Spectrum Overview, Appl. Phys. Rev. 7, 021309 (2020).

[7] N. Somaschi et al., Near-Optimal Single-Photon Sources in the Solid State, Nat. Photonics 10, 340 (2016).

[8] L. Schweickert et al., On-Demand Generation of Background-Free Single Photons from a Solid-State Source, Appl. Phys. Lett. 112, 093106 (2018).

[9] L. Childress and R. Hanson, Diamond NV Centers for Quantum Computing and Quantum Networks, MRS Bull. 38, 134 (2013).

[10] L. Robledo, L. Childress, H. Bernien, B. Hensen, P. F. A. Alkemade, and R. Hanson, High-Fidelity Projective ReadOut of a Solid-State Spin Quantum Register, Nature (London) 477, 574 (2011).

[11] R. Vasconcelos, S. Reisenbauer, C. Salter, G. Wachter, D. Wirtitsch, J. Schmiedmayer, P. Walther, and M. Trupke, Scalable Spin-Photon Entanglement by Time-to-Polarization Conversion, npj Quantum Inf. 6, 9 (2020).

[12] S. Kumar, M. Brotóns-Gisbert, R. Al-Khuzheyri, A. Branny, G. Ballesteros-Garcia, J. F. Sánchez-Royo, and B. D. Gerardot, Resonant Laser Spectroscopy of Localized Excitons in Monolayer WSe 2, Optica 3, 882 (2016).

[13] P. Siyushev et al., Coherent Properties of Single Rare-Earth Spin Qubits, Nat. Commun. 5, 3895 (2014).

[14] M. E. Trusheim et al., Transform-Limited Photons from a Coherent Tin-Vacancy Spin in Diamond, Phys. Rev. Lett. 124, 023602 (2020).

[15] T. Müller, C. Hepp, B. Pingault, E. Neu, S. Gsell, M. Schreck, H. Sternschulte, D. Steinmüller-Nethl, C. Becher, and M. Atatüre, Optical Signatures of Silicon-Vacancy Spins in Diamond, Nat. Commun. 5, 3328 (2014).

[16] D. Chen, Z. Mu, Yu. Zhou, J. E. Fröch, A. Rasmit, C. Diederichs, N. Zheludev, I. Aharonovich, and W.-b. Gao, Optical Switching of Resonance Fluorescence from a Single Germanium Vacancy Color Center in Diamond, Phys. Rev. Lett. 123, 033602 (2019).
[17] A. N. Vamivakas, Y. Zhao, C.-Y. Lu, and M. Atatüre, SpinResolved Quantum-Dot Resonance Fluorescence, Nat. Phys. 5, 198 (2009).

[18] T. Kaldewey, A. V. Kuhlmann, S. R. Valentin, A. Ludwig, A. D. Wieck, and R. J. Warburton, Far-Field Nanoscopy on a Semiconductor Quantum Dot via a Rapid-AdiabaticPassage-Based Switch, Nat. Photonics 12, 68 (2018).

[19] H.-S. Nguyen, G. Sallen, C. Voisin, Ph. Roussignol, C. Diederichs, and G. Cassabois, Ultra-coherent Single Photon Source, Appl. Phys. Lett. 99, 261904 (2011).

[20] D. Najer et al., A Gated Quantum Dot Strongly Coupled to an Optical Microcavity, Nature (London) 575, 622 (2019).

[21] A. K. Nowak, S. L. Portalupi, V. Giesz, O. Gazzano, C. Dal Savio, P.-F. Braun, K. Karrai, C. Arnold, L. Lanco, I. Sagnes, A. Lemaître, and P. Senellart, Deterministic and Electrically Tunable Bright Single-Photon Source, Nat. Commun. 5, 3240 (2014).

[22] M. Müller, S. Bounouar, K. D. Jöns, M. Glässl, and P. Michler, On-Demand Generation of Indistinguishable Polarization-Entangled Photon Pairs, Nat. Photonics 8, 224 (2014).

[23] E. Schöll et al., Resonance Fluorescence of GaAs Quantum Dots with Near-Unity Photon Indistinguishability, Nano Lett. 19, 2404 (2019).

[24] A. V. Kuhlmann, J. Houel, A. Ludwig, L. Greuter, D. Reuter, A. D. Wieck, M. Poggio, and R. J. Warburton, Charge Noise and Spin Noise in a Semiconductor Quantum Device, Nat. Phys. 9, 570 (2013).

[25] H. Ollivier et al., Reproducibility of High-Performance Quantum Dot Single-Photon Sources, ACS Photonics 7, 1050 (2020).

[26] A. Tchebotareva et al., Entanglement between a Diamond Spin Qubit and a Photonic Time-Bin Qubit at Telecom Wavelength, Phys. Rev. Lett. 123, 063601 (2019).

[27] N. Tomm et al., A Bright and Fast Source of Coherent Single Photons, arXiv:2007.12654 [Nat. Nanotechnol. (to be published)], https://www.nature.com/articles/s41565-02000831-x.

[28] R. H. Jensen et al., Cavity-Enhanced Photon Emission from a Single Germanium-Vacancy Center in a Diamond Membrane, Phys. Rev. Applied 13, 064016 (2020).

[29] T. Wilson and R. Juškaitis, On the Extinction Coefficient in Confocal Polarization Microscopy, J. Microscopy 179, 238 (1995).

[30] P. Higdon, R. Juškaitis, and T. Wilson, The Effect of Detector Size on the Extinction Coefficient in Confocal Polarization Microscopes, J. Microscopy 187, 8 (1997).

[31] A. V. Kuhlmann et al., A Dark-Field Microscope for Background-Free Detection of Resonance Fluorescence from Single Semiconductor Quantum Dots Operating in a Set-and-Forget Mode, Rev. Sci. Instrum. 84, 073905 (2013).

[32] F. I. Fedorov, On the Theory of Total Reflection, Dokl. Akad. Nauk SSSR 105, 465 (1955).

[33] C. Imbert, Calculation and Experimental Proof of the Transverse Shift Induced by Total Internal Reflection of a Circularly Polarized Light Beam, Phys. Rev. D 5, 787 (1972).

[34] T. Ozawa et al., Topological Photonics, Rev. Mod. Phys. 91, 015006 (2019). 
[35] V. S. Liberman and B. Y. Zel'dovich, Spin Orbit Interaction of a Photon in an Inhomogeneous Medium, Phys. Rev. A 46, 5199 (1992).

[36] K. Y. Bliokh, F. J. Rodríguez-Fortuño, F. Nori, and A. V. Zayats, Spin-Orbit Interactions of Light, Nat. Photonics $\mathbf{9}$, 796 (2015).

[37] F. Stern, Elementary Theory of the Optical Properties of Solids, in Solid State Physics (Elsevier, New York, 1963), Vol. 15, pp. 299-408.

[38] P. B. Johnson and R.-W. Christy, Optical Constants of the Noble Metals, Phys. Rev. B 6, 4370 (1972).

[39] L. Novotny and B. Hecht, Principles of Nano-Optics (Cambridge University Press, Cambridge, England, 2012).

[40] A. Aiello and H. Woerdman, The Reflection of a MaxwellGaussian Beam by a Planar Surface, arXiv:0710.1643.

[41] F. Goos and H. Hänchen, Ein Neuer und Fundamentaler versuch zur Totalreflexion, Ann. Phys. (Berlin) 436, 333 (1947).

[42] M. Onoda, S. Murakami, and N. Nagaosa, Hall Effect of Light, Phys. Rev. Lett. 93, 083901 (2004).

[43] A. Aiello and J. Woerdman, Role of Beam Propagation in Goos-Hänchen and Imbert-Fedorov Shifts, Opt. Lett. 33, 1437 (2008).

[44] J. W. Goodman, Introduction to Fourier Optics (McGrawHill Book Comp., New York, 1968).

[45] Y. Aharonov, D. Z. Albert, and L. Vaidman, How the Result of a Measurement of a Component of the Spin of a Spin-1/2
Particle Can Turn out to Be 100, Phys. Rev. Lett. 60, 1351 (1988).

[46] I. Duck, P. M. Stevenson, and E. Sudarshan, The Sense in which a "Weak Measurement" of a Spin-1/2 Particle's Spin Component Yields a Value 100, Phys. Rev. D 40, 2112 (1989).

[47] O. Hosten and P. Kwiat, Observation of the Spin Hall Effect of Light via Weak Measurements, Science 319, 787 (2008).

[48] W. L. Erikson and S. Singh, Polarization Properties of Maxwell-Gaussian Laser Beams, Phys. Rev. E 49, 5778 (1994).

[49] X. Hong, E. M. P. H. van Dijk, S. R. Hall, J. B. Götte, N. F. van Hulst, and H. Gersen, Background-Free Detection of Single $5 \mathrm{~nm}$ Nanoparticles through Interferometric CrossPolarization Microscopy, Nano Lett. 11, 541 (2011).

[50] H. B. Shim and J. W. Hahn, Plasmonic Near-Field Scanning Nanoscope with a Cross-Polarization Detection Technique, Nanophotonics 8, 1731 (2019).

[51] J.-P. Kakko, A. Matikainen, N. Anttu, S. Kujala, H. Mäntynen, V. Khayrudinov, A. Autere, Z. Sun, and H. Lipsanen, Measurement of Nanowire Optical Modes Using Cross-Polarization Microscopy, Sci. Rep. 7, 17790 (2017).

[52] G. Wrigge, I. Gerhardt, J. Hwang, G. Zumofen, and V. Sandoghdar, Efficient Coupling of Photons to a Single Molecule and the Observation of Its Resonance Fluorescence, Nat. Phys. 4, 60 (2008). 